\title{
Angular momentum transport and element mixing in the stellar interior
}

\section{Application to the rotating Sun}

\author{
W. M. Yang ${ }^{1,2}$ and S. L. Bi ${ }^{1}$ \\ 1 National Astronomical Observatories/Yunnan Observatory, Chinese Academy of Sciences, Kunming 650011, PR China \\ e-mail: yangwuming@ynao.ac.cn; bislan@public.km.yn.cn \\ 2 Graduate School of The Chinese Academy of Sciences, Beijing 100039, PR China
}

Received 6 June 2005 / Accepted 15 November 2005

\section{ABSTRACT}

Aims. The purpose of this work was to obtain diffusion coefficient for the magnetic angular momentum transport and material transport in a rotating solar model.

Methods. We assumed that the transport of both angular momentum and chemical elements caused by magnetic fields could be treated as a diffusion process.

Results. The diffusion coefficient depends on the stellar radius, angular velocity, and the configuration of magnetic fields. By using of this coefficient, it is found that our model becomes more consistent with the helioseismic results of total angular momentum, angular momentum density, and the rotation rate in a radiative region than the one without magnetic fields. Not only can the magnetic fields redistribute angular momentum efficiently, but they can also strengthen the coupling between the radiative and convective zones. As a result, the sharp gradient of the rotation rate is reduced at the bottom of the convective zone. The thickness of the layer of sharp radial change in the rotation rate is about $0.036 R_{\odot}$ in our model. Furthermore, the difference of the sound-speed square between the seismic Sun and the model is improved by mixing the material that is associated with angular momentum transport.

Key words. Sun: rotation - Sun: magnetic fields - diffusion

\section{Introduction}

In standard stellar models, it is assumed that there are no rotation and magnetic fields. Although this framework is strongly supported by helioseismology, recent advances in the study of solar structure shows that differences exist between the Sun and this model. Those differences are small and display a very interesting systematic behavior, which is far from satisfactory (Christensen-Dalsgaard 2002). The most striking difference is the bump in the sound speed just beneath the convection zone. Noting both $c^{2} \propto T / \mu$ and the $\mu$-gradient caused by microscopic diffusion and element settling in the radiative region, Gough et al. (1996) and Christensen-Dalsgaard et al. (1996, 2002) argued that material mixing may be important but has been neglected.

Helioseimology also shows that rotation is almost uniform in the solar radiative region: the Sun has a slow rotation core, the angular velocity $\Omega$ has a small radial gradient and large latitudinal gradient in the convective zone, and the radial angular velocity gradient is positive at low latitudes and negative at high latitudes in the tachocline (Schou et al. 1998; Chaplin et al. 1999). But the calculations of rotational models show that the sun has a fast rotation core and a large gradient of the rotation rate in the radiative region (Pinsonneault et al. 1989; Charboyer et al. 1995), which disagrees with the helioseismic results. Thompson et al. (2003) argues that some mechanisms of angular momentum transport may be missed in these models. Magnetic and gravity waves mechanisms have been proposed; however, what mechanisms are causing the nearly uniform rotation in the radiative region are poorly understood.

Rotation is a property that virtually all stars possess. Rotation affects stellar structure mainly in two ways. The first is an immediately dynamical effect caused by centrifugal acceleration of the hydrostatic balance. In most cases, however, this effect is modest and can be included through modifying the equations of stellar structure. Although the equations of stellar structure of a rotating star are three-dimensional, the method of Kippenhahn \& Thomas (1970), which uses the mass contained within an equipotential surface, $M_{\Psi}$, as an 
independent variable, allows a one-dimensional evolution code to be modified to incorporate the hydrostatic effects of rotation. This method was modified by Endal \& Sofia (1976) and Meynet \& Maeder (1997) to apply to shellular rotation (Zahn 1992), a rotation rate that depends only on the radial coordinate $r$ to a first approximation. The other way is an effect arising from the redistribution of chemical elements due to the instabilities caused by rotation. This effect is much more important and it has been studied by many investigators (Endal \& Sofia 1978; Pinsonneault et al. 1989; Chaboyer \& Zahn 1992; Zahn 1992; Meynet \& Maeder 1997; Maeder 1997; Maeder \& Zahn 1998; Maeder \& Meynet 2000; Huang 2004a, 2004b). The method of Kippenhahn \& Thomas (1970) as modified by Meynet \& Maeder (1997) is used in our model.

Magnetic fields are another property of stars. Their effects on a star create a complicated problem. The magnetic field is involved in most problems in astrophysics. But the generation of magnetic fields is still a controversial problem. They may be fossil fields (Cowling 1945; Moss 1987; Braithwaite 2004), which are remnants of the star's formation, or may be generated by a convective stellar dynamo (Parker 1979; Charbonneau \& MacGregor 2001) in the convective zone or generated by a Tayler-Spruit dynamo (Pitts \& Tayler 1986; Spuit 2002) in a differential rotation star. In this paper we assumed that the magnetic fields exist. We only consider the process of magnetic angular momentum transport and material mixing. Although the magnetic angular momentum transport has been treated by many investigators (Charbonneau \& MacGregor 1992, 1993; Spruit 2002; Maeder \& Meynet 2003, 2004), it is still an open question.

In this paper, we focus mainly on magnetic angular momentum transport and the mixing of elements associated with angular momentum redistribution. In Sect. 2 we give the equations of stellar structure of a shellular rotation star. In Sect. 3 we deduce the diffusion coefficient for magnetic angular momentum transport and material mixing that is due to angular momentum redistribution. Then, in Sect. 4 we give the results of the numerical calculation. We then discuss our results and conclude in Sect. 5.

\section{Equations of stellar structure of a shellular rotation star}

The stars whose angular velocity is constant on their isobars are called shellular rotation stars (Meynet \& Maeder 1997); the isobar surfaces are given by (Meynet \& Maeder 1997)

$\Psi_{p}=\Phi+\frac{1}{2} \Omega^{2} r^{2} \sin ^{2} \theta=$ const.

where $\Phi$ is the minus gravitational potential, $\Omega$ the angular velocity, $r$ the radius, and $\theta$ the colatitude. The area of such an isobar surface is denoted by $S_{p}$, and the volume enclosed by the isobar surface by $V_{p}$. For any quantity $q$, which is not constant over an isobar surface, a mean value is defined by

$\langle q\rangle=\frac{1}{S_{p}} \int_{\psi=\text { const. }} q \mathrm{~d} \sigma$,

where $\mathrm{d} \sigma$ is an element of the isobar surface.
The equations of stellar structure of a shellular rotation star, which were developed by Kippenhahn \& Thomas (1970) and Meynet \& Maeder (1997), are as follows:

$$
\begin{aligned}
\frac{\partial P}{\partial M_{p}} & =-\frac{G M_{p}}{4 \pi r_{p}^{4}} f_{p}, \\
\frac{\partial r_{p}}{\partial M_{p}} & =\frac{1}{4 \pi r_{p}^{2} \bar{\rho}} \\
\frac{\partial L_{p}}{\partial M_{p}} & =\epsilon_{n}-\epsilon_{v}+\epsilon_{g}, \\
\frac{\partial T}{\partial M_{p}} & =-\frac{G M_{p}}{4 \pi r_{p}^{4}} f_{p} \min \left[\nabla_{\mathrm{ad}}, \nabla_{\mathrm{rad}} \frac{f_{T}}{f_{p}}\right]
\end{aligned}
$$

where $r_{p}$ is the radius of a sphere enclosing the volume $V_{p}$, i.e.,

$V_{p}=\frac{4 \pi}{3} r_{p}^{3}$

The $M_{p}$ is the mass inside the isobar, and

$$
\begin{aligned}
& f_{p}=\frac{4 \pi r_{p}^{4}}{G M_{p} S_{p}} \frac{1}{\left\langle g_{\mathrm{e}}^{-1}\right\rangle} \\
& \bar{\rho}=\frac{\rho\left(1-r^{2} \sin ^{2} \theta \Omega \alpha\right)\left\langle g_{\mathrm{e}}^{-1}\right\rangle}{\left\langle g_{\mathrm{e}}^{-1}\right\rangle-\left\langle g_{\mathrm{e}}^{-1} r^{2} \sin ^{2}\right\rangle \Omega \alpha} \\
& f_{T}=\left(\frac{4 \pi r_{p}^{2}}{S_{p}}\right) \frac{1}{\left\langle g_{\mathrm{e}}\right\rangle\left\langle g_{\mathrm{e}}^{-1}\right\rangle}
\end{aligned}
$$

Here $g_{\mathrm{e}}$ is the effective gravity. The $\alpha$ in Eq. (9) is a scalar $\frac{\mathrm{d} \Omega}{\mathrm{d} \Psi}$. Equation (3) is the hydrostatic equilibrium equation including centrifugal force, while Eq. (6) is the equation of energy transport under the effects of rotation. The nondimensional rotating corrective factors $f_{p}$ and $f_{T}$ depend on the shape of the isobars.

Assuming that the shapes of isobars are spheroids with semi-major axis $a$ and semi-minor axis $b$, and given the angular velocity distribution, using definitions (7) and (1), i.e.,

$\frac{4}{3} \pi a^{2} b=\frac{4}{3} \pi r_{p}^{3}$

$\frac{G M_{p}}{b}=\frac{G M_{p}}{a}+\frac{1}{2} \Omega^{2} a^{2}$,

we can get the values of $a$ and $b$ for any given $M_{p}$ and $r_{p}$; i.e., the surface of the isobar is determined. Thus, the average values of the effective gravity and its inverse $\left(\left\langle g_{\mathrm{e}}\right\rangle\right.$ and $\left.\left\langle g_{\mathrm{e}}^{-1}\right\rangle\right)$ can be obtained. Then the $f_{p}$ and $f_{T}$ are also obtained. In the computation, the Roche mode (Kippenhahn \& Thomas 1990) was used to compute the gravitational potential. 


\section{Angular momentum transport and the mixing of elements}

\subsection{Hydrodynamical instabilities}

The mechanisms that redistribute angular momentum and the chemical elements in a rotating star can be divided into two categories according to the time scale involved.

The first category is that of the dynamical instabilities, occurring on the dynamical timescale. If the dynamical unstable gradient occurs in star, it can be instantaneously smoothed (Endal \& Sofia 1978). One of the dynamical instabilities is the convective instability. We suppose that solid-body rotation was enforced in all convective regions. Another much more important is dynamical shear instabilities. These instabilities can ensure that the rotation velocity is constant on equipotential surfaces (Pinsonneault et al. 1989).

The second category, with a time scale that is comparable to the Kelvin-Helmholtz time scale or the time scale for the evolution of the star, is that of secular instabilities. For secular instabilities, the transport process of angular momentum and chemical composition was treated as a diffusion process (Endal \& Sofia 1978; Pinsonneault et al. 1989), so the radial equations for the redistribution of angular momentum and for the mass fraction $X_{i}$ are

$$
\begin{aligned}
& \frac{\partial \Omega}{\partial t}= f_{\Omega} \frac{1}{\rho r^{4}} \frac{\partial}{\partial r}\left(\rho r^{4} D_{d} \frac{\partial \Omega}{\partial r}\right)-\frac{1}{\rho r^{4}} \frac{\partial}{\partial r}\left(\rho r^{4} \Omega \dot{r}\right), \\
& \frac{\partial X_{i}}{\partial t}= f_{c} f_{\Omega} \frac{1}{\rho r^{2}} \frac{\partial}{\partial r}\left(\rho r^{2} D_{d} \frac{\partial X_{i}}{\partial r}\right) \\
&+\left(\frac{\partial X_{i}}{\partial t}\right)_{\mathrm{nuc}}-\frac{1}{\rho r^{2}} \frac{\partial}{\partial r}\left(\rho r^{2} X_{i} V_{i}\right),
\end{aligned}
$$

where $D_{\mathrm{d}}$ is the diffusion coefficient. Because of some inherent uncertainties in the diffusion equation, the adjustable parameter $f_{\Omega}$ is introduced to represent these uncertainties. Another adjustable parameter $f_{c}$ is used to account for how the instabilities mix material less efficiently than they transport angular momentum (Pinsonneault et al. 1989). The second term on the right-side of Eq. (13) is due to the secular contraction and/or expansion (Maeder \& Zahn 1998), which can be dominant to induce differential rotation in a rotating star with a weak wind or without any wind. The second term on the right-side of Eq. (14) is the change in the nuclear reaction. The $V_{i}$ in the Eq. (14) is the velocity of microscopic diffusion given by Thoul et al. (1994). In our model, we use the diffusion coefficient that was given by Zahn (1993) for secular shear instability,

$D_{\mathrm{d}}=\frac{2 c}{27 G}\left|\frac{\mathrm{d} \ln T}{\mathrm{~d} r}-\frac{2}{3} \frac{\mathrm{d} \ln \rho}{\mathrm{d} r}\right|^{-1} \frac{r^{4}}{\kappa \rho M(r)}\left(\frac{\mathrm{d} \Omega}{\mathrm{d} r}\right)^{2}$.

It has been thought that the Sun and T-Tauri with about one solar mass both possess an initial angular momentum of the order of $10^{50} \mathrm{~g} \mathrm{~cm}^{2} \mathrm{~s}^{-1}$ (Kawaler 1987; Paternò 1991). The present total solar angular momentum is about $10^{48} \mathrm{~g} \mathrm{~cm}^{2} \mathrm{~s}^{-1}$ (Pijpers 1998; Antia et al. 2000; Komm et al. 2003). Thus, the mean angular momentum loss rate is not less than $10^{40} \mathrm{~g} \mathrm{~cm}^{2} \mathrm{~s}^{-1} \mathrm{yr}^{-1}$.
Using the formula for angular momentum loss (Mestel 1984; Kawaler 1988),

$$
\frac{\mathrm{d} J}{\mathrm{~d} t}=\frac{2}{3} \frac{\mathrm{d} M}{\mathrm{~d} t} R^{2} \Omega_{\mathrm{s}}\left(\frac{r_{\mathrm{A}}}{R}\right)^{2},
$$

and adopting the values of $\frac{\mathrm{d} J}{\mathrm{~d} t}, \frac{\mathrm{d} M}{\mathrm{~d} t}$, and $\Omega_{\mathrm{s}}$ as $10^{40} \mathrm{~g} \mathrm{~cm}^{2} \mathrm{~s}^{-1} \mathrm{yr}^{-1}, 10^{-14} M_{\odot} \mathrm{yr}^{-1}$, and $10^{-5} \mathrm{rad} \mathrm{s}^{-1}$, respectively, we can get the corotational radius $r_{\mathrm{A}}$ of solar wind is $100 R_{\odot}$. It seems difficult for the solar wind to remain corotation with the Sun at a distance of $100 R_{\odot}$. In this paper, we adopt a low initial angular momentum and ignore solar magnetic braking at the surface. This situation was also studied by Eggenberger et al. (2005). Circulation is ignored because the circulation is very weak in a no-wind star, or it can even vanish in slow rotators (Zahn 1992). In our models, the total angular momentum is conserved, and the secular contraction and/or expansion are dominant to induce interior differential rotation.

\subsection{Magnetic effects}

Spruit (1999) reviewed the known magnetic instabilities in differentially rotating, stably stratified stellar interiors. Recently, Spruit $(1999,2002)$ and Maeder \& Meynet $(2003,2004)$ developed the Tayler-Spruit dynamo theory, which can generate magnetic fields in the radiative interior of differentially rotating stars. These fields are predominantly azimuthal components, $B \sim B_{\phi}$. The existence of magnetic field and magnetic instabilities in the stellar interior provides a process in which angular momentum and chemical compositions can be transported by magnetic stresses and magnetic instabilities. In this paper, we have assumed that the transport of angular momentum and mixing of material can be treated as a diffusion process. In the following, we give the diffusion coefficient for this process.

In the plasma, the magnetic Prandtl number is (Brandenburg \& Subramanian 2005)

$P_{\mathrm{m}} \equiv \frac{\nu}{\eta}=1.1 \times 10^{-4}\left(\frac{T}{10^{6}}\right)^{4}\left(\frac{\rho}{0.1 \mathrm{~g} \mathrm{~cm}^{-3}}\right)^{-1}\left(\frac{\ln \Lambda}{20}\right)^{-2}$,

where $v$ is the kinematic viscosity, $\eta$ the magnetic diffusivity, and the $\ln \Lambda$ is the coulomb logarithm. In the solar radiative region $\left(T \sim 8 \times 10^{6}-2 \times 10^{6} \mathrm{~K}, \rho \sim 20-0.2 \mathrm{~g} \mathrm{~cm}^{-3}\right)$, the $P_{\mathrm{m}}$ is about $10^{-2}-10^{-3}$. The $P_{\mathrm{m}}$ is about $10^{-4}-10^{-7}$ in the solar convective zone (Brandenburg \& Subramanian 2005). We assume $\ln \Lambda=10$ in the above calculation. Thus, we ignore viscosity in the following equation. For a constant magnetic diffusivity and shellular rotation, under axisymmetry and only considering Lorentz force, the azimuthal components of the induction and momentum equations are

$\frac{\partial B_{\phi}}{\partial t}+\eta\left(\frac{1}{r^{2} \sin ^{2} \theta}-\nabla^{2}\right) B_{\phi}=r \sin \theta B_{r} \frac{\partial \Omega}{\partial r}$,

$\frac{\partial \Omega}{\partial t}=\frac{1}{4 \pi \rho r^{2} \sin ^{2} \theta} \mathbf{B}_{p} \cdot \nabla\left(r \sin \theta B_{\phi}\right)$.

If we assume that the effect of the magnetic diffusivity is to limit the growth of the toroidal field after some time (Mestel 
et al. 1987), the growth of the instability is halted by dissipative processes that operate on a timescale $\tau$, so that the second term on the left-hand side of Eq. (18) may be replaced simply by $B_{\phi} / \tau$ (Barnes et al. 1999). Substituting for the second term of Eq. (18) and differentiating the Eq. (18) with respect to time, we obtain (Barnes et al. 1999)

$$
\frac{\partial^{2} B_{\phi}}{\partial^{2} t}+\frac{1}{\tau} \frac{\partial B_{\phi}}{\partial t}=r \sin \theta \mathbf{B}_{r} \cdot \nabla \frac{\partial \Omega}{\partial t}
$$

For much longer times than the timescale of the instability, one would expect the term involving the first time derivative to dominate, so that (Barnes et al. 1999)

$$
\begin{aligned}
\frac{\partial\left(r \sin \theta B_{\phi}\right)}{\partial t} \simeq & \tau(r \sin \theta)^{2} \mathbf{B}_{r} \cdot \nabla\left[\frac{1}{4 \pi \rho(r \sin \theta)^{2}} \mathbf{B}_{p}\right. \\
& \left.\cdot \nabla\left(r \sin \theta B_{\phi}\right)\right] \\
\approx & \nabla \cdot\left[D_{\mathrm{m}} \nabla\left(r \sin \theta B_{\phi}\right)\right] .
\end{aligned}
$$

Equation (21) is an approximate diffusion equation for $r \sin \theta B_{\phi}$ with a diffusion coefficient

$D_{\mathrm{m}}=\frac{\tau B_{r}^{2}}{4 \pi \rho}$

For a steady equilibrium, the dissipating timescale has to match the growth timescale of the instability. In rotating stars under the condition $\omega_{\mathrm{A}} \ll \Omega$, the growth time scale of the magnetic instability is (Pitts \& Tayler 1986; Spruit 1999)

$\sigma^{-1}=\frac{\Omega}{\omega_{\mathrm{A}}^{2}}$,

where

$\omega_{\mathrm{A}}=\frac{B}{(4 \pi \rho)^{1 / 2} r}$,

is the Alfvén frequency. If $\tau=\sigma^{-1}$, one can get

$$
\begin{aligned}
D_{\mathrm{m}} & =\frac{B_{r}^{2}}{4 \pi \rho} \frac{\Omega}{\omega_{\mathrm{A}}^{2}} \\
& =r^{2} \Omega \frac{B_{r}^{2}}{B^{2}} .
\end{aligned}
$$

Note the diffusion coefficient $D_{\mathrm{m}}$ derived from the induction and momentum equations, so that one would expect that the angular momentum transport and the mixing of elements caused by magnetic fields obey a similar diffusion equation, such as Eqs. (13) and (14) with the diffusion coefficient $D_{\mathrm{m}}$. Although this diffusion coefficient is used in the equations of angular momentum transport and the mixing of elements, which is only an assumption, expression (25) hints that this diffusion coefficient is related to the efficiency of the angular momentum transport. Expression (25) can be rewritten as

$D_{\mathrm{m}}=r^{2} \Omega \frac{v_{r \mathrm{~A}}^{2}}{v_{\mathrm{A}}^{2}}$,

where

$v_{r \mathrm{~A}}^{2}=\frac{B_{r}^{2}}{4 \pi \rho}$

$v_{\mathrm{A}}^{2}=\frac{B^{2}}{4 \pi \rho}$,

is the Alfvén velocity square. If the angular momentum is transported by an Alfvén wave, and the magnetic field $B$ dominates as an azimuthal field, so that the larger the ratio $v_{r \mathrm{~A}}^{2} / v_{\mathrm{A}}^{2}$, the more efficient the angular momentum transport.

\section{Numerical calculation and results}

\subsection{Choice of parameters}

The code originally written by Paczyński $(1969,1970)$ was updated by Sienkiewicz in 1995 and Yang et al. (2001). We modified it to incorporate the hydrostatic effects of rotation on the equations of stellar structure, using the method of Kippenhahn-Meynet (Kippenhahn \& Thomas 1970; Meynet \& Maeder 1997). The models are calculated using the OPAL equation of state (Rogers et al. 1996), OPAL opacity (Iglesias \& Rogers 1996), and the Alexander \& Ferguson (1994) opacity table for low temperature. Element diffusion is incorporated for helium and metals (Thoul et al. 1994). The nuclear reaction rates have been updated according to Bahcall \& Pinsonneault (1995). Energy transfer by convection is treated according to the standard mixing length theory, and the boundaries of the convection zones are determined by the Schwarzschild criterion. We adopt the solar age as $4.6 \times 10^{9}$ year, luminosity $L_{\odot}=3.844 \times 10^{33} \mathrm{erg} \mathrm{s}^{-1}$, radius $R_{\odot}=6.96 \times 10^{10} \mathrm{~cm}$, and the ratio of heavy elements to hydrogen by mass $Z / X=0.023$ (Grevesse \& Sauval 1998).

As mentioned above, we assume that solid body rotation was enforced in the convective region. This assumption has been used by Pinsonneault et al. (1989), Chaboyer et al. (1995), and Huang (2004b). The total initial angular momentum is a free parameter that is adjusted until the surface velocities of the solar-age models are near the solar surface velocity. We take the rotation rate of the Sun as a solid-body rotation, about $2.72 \times 10^{-6} \mathrm{rad} / \mathrm{s}$ (Komm et al. 2003), as the rotation rate of convective zone of our model.

The strength and spatiotemporal distribution of magnetic fields inside the star are poorly known. Classical dynamo models predict toroidal fields that are not stronger than about $10^{4}$ Gauss. But Choudhuri \& Gilman (1987), D’Silva \& Choudhuri (1993), and Caligari et al. (1995) have pointed out the value of the magnetic field at the bottom of convective zone as around $10^{5}$ Gauss. The virial theorem (Parker 1979) sets an upper limit to the magnitude of the average solar magnetic field: $\langle B\rangle \leq 10^{8}$ Gauss. Dudorov et al. (1989) estimated the value of the poloidal magnetic field in the solar radiative zone to be about the order of unity. Using a kinematic model with prescribed internal rotation and a standard solar model, Fox and Bernstein (1987) investigated the existence of large-scale magnetic fields in the Sun, and found that the ratio of poloidal to toroidal components of the magnetic field is about $10^{-10}$. Although the ratio of $B_{r}$ to $B$ may change with the radial coordinate $r$, the rotation rate $\Omega$, and the time $t$, the relation of $\frac{B_{r}}{B}$ to $r, \Omega$, and $t$ is unclear. As a first test, we take $B_{r} / B$ to be a constant, and take the order of the ratio of $B_{r}$ to $B$ as $10^{-5}$ in the radiative region.

In order to study the effects of magnetic fields, we construct three different types of solar models. These models are labelled as follows:

- M1: model with no rotation and no magnetic field but with element diffusion;

- M2: model with rotation and no magnetic field but with element diffusion; 
Table 1. Model parameters.

\begin{tabular}{cccc}
\hline \hline Parameter & M1 & M2 & M3 \\
\hline$\alpha$ & 1.797 & 1.790 & 1.790 \\
$Y_{0}$ & 0.2708 & 0.2707 & 0.2707 \\
$(Z / X)_{0}$ & 0.0276 & 0.0277 & 0.0277 \\
$Y_{\mathrm{s}}$ & 0.2437 & 0.2439 & 0.2441 \\
$(Z / X)_{\mathrm{s}}$ & 0.023 & 0.0231 & 0.0232 \\
$R_{b c z} / R_{\odot}$ & 0.709 & 0.710 & 0.711 \\
$f_{\Omega}$ & & 1.0 & $6.5 \times 10^{-3}$ \\
$f_{\mathrm{c}}$ & & 0.02 & 0.03 \\
\hline
\end{tabular}

Note. $\alpha$ is the mixing-length parameter; $Y_{0}$ and $(Z / X)_{0}$ are the initial chemical compositions; $Y_{\mathrm{s}}$ and $(Z / X)_{s}$ are the surface compositions at an age of $4.6 \times 10^{9}$ years; $R_{b c z}$ is the radius at the base of the convective zone.

- M3: model with rotation and a magnetic field and element diffusion, but without including the effects of secular shear instability.

Some parameters are summarized in Table 1. The mixinglength parameters $\alpha, Z_{0}, X_{0}, f_{\Omega}$, and $f_{\mathrm{c}}$ are free parameters. The $X_{0}, Z_{0}$ and $\alpha$ are adjusted until the solar-age model has the values of the solar luminosity, radius, and $(Z / X)_{\mathrm{s}}$. Then $Y_{0}$ is determined by $Y_{0}=1-X_{0}-Z_{0}$. The parameter $f_{\Omega}$ was adjusted to fit the radial profiles of the angular velocity, which were got from helioseismology in the solar interior. Parameter $f_{\mathrm{c}}$ was adjusted to get the best radial profile of the sound speed. Finally, $Y_{\mathrm{s}},(Z / X)_{\mathrm{s}}$ and $R_{b c z}$ are the results of calculation. All models are constructed by evolving a fully convective, pre-main-sequence, one solar mass model to the age of the present Sun.

\subsection{Transport of angular momentum}

We define the angular momentum density $J(r, t)$ and total angular momentum $A(t)$ as

$J(r, t)=\frac{8 \pi}{3} \rho r^{4} \Omega(r, t)$,

$A(t)=\int_{0}^{R_{\odot}} J(r, t) \mathrm{d} r$,

where $t$ is time.

In Figs. 1a and b, we present the rotation rates and their differentiation with respect to radius $r$ as a function $r$ at the age of $4.6 \mathrm{G}$ years. The radial profile of the rotation rate of model M3 is flatter than of model M2. In our models, the radial gradient of angular velocity is negative. But the helioseismology has revealed that the gradient is positive at a low latitude and negative at a high latitude at the bottom of the solar convective zone (Schou et al. 1998). At around $r=0.7 R_{\odot}$ in models M2 and M3, a sharp radial change in the rotation curve takes place. The thickness of the layer of sharp radial change in the rotation rate is about $0.036 R_{\odot}$. Helioseismoloy also shows that that layer exists, and the thickness of the layer is around between $0.02 R_{\odot}$ and $0.05 R_{\odot}$ (Christensen-Dalsgaard et al. 1991; Kosovichev 1996; Basu \& Antia 1997a,b; Corbard et al. 1998; Corbard et al. 1999; Charbonneau et al. 1999). The rotation rate of model M3 is lower for $r<0.4 R_{\odot}$ and higher for $r>0.4 R_{\odot}$ than for model M2, which has the same total angular momentum as model M3. When M2 and M3 have the same surface rotation rate, the rotation rate of M2 is higher than it is for M3 in whole radiative region.

The diffusion coefficient of hydrodynamical model $D_{\mathrm{d}}$ is very small in the inner portion of the radiative region. It is ineffective to transport angular momentum outwards. But the diffusion coefficient of magnetic model $D_{\mathrm{m}}$ is large enough to transport angular momentum from the core to the convective zone. That is to say magnetic fields are more efficient than the secular shear instabilities in transport angular momentum. The magnetic fields not only transport angular momentum outwards but strengthen the coupling between the radiative and convective zone. Thus, under with the same total angular momentum, the rotation rate of model M3 is lower for $r<0.4 R_{\odot}$ but is higher for $r>0.4 R_{\odot}$ than the one for model M2 (Fig. 1a); the gradient of the rotation rate of model M3 is significantly reduced beneath the convective zone (Fig. 1b).

The angular momentum density $J$ and its differentiation with respect to $r$ of models M2 and M3, both with the same surface rotation rate at the age of $4.6 \mathrm{Gyr}$, are shown in Figs. 1c and d. The total angular momentum of model M3 is $1.91 \times 10^{48} \mathrm{~g} \mathrm{~cm}^{2} \mathrm{~g}^{-1}$, which is consistent with helioseismic results $(1.900 \pm 0.0015) \times 10^{48} \mathrm{~g} \mathrm{~cm}^{2} \mathrm{~s}^{-1}$ (Pijpers 1998), $1.91 \times 10^{48} \mathrm{~g} \mathrm{~cm}^{2} \mathrm{~s}^{-1}$ (Antia et al. 2000), or $(1.94 \pm 0.05) \times$ $10^{48} \mathrm{~g} \mathrm{~cm}^{2} \mathrm{~s}^{-1}$ (Komm et al. 2003), but that of model M2 is $2.1 \times 10^{48} \mathrm{~g} \mathrm{~cm}^{2} \mathrm{~g}^{-1}$, which is higher than helioseismic results. The angular momentum density $J$ shows a maximum $5.51 \times 10^{37} \mathrm{~g} \mathrm{~cm} \mathrm{~s}^{-1}$ at $r=0.35 R_{\odot}$ of model M3, and $6.1 \times$ $10^{37} \mathrm{~g} \mathrm{~cm} \mathrm{~s}^{-1}$ at $r=0.34 R_{\odot}$ of model M2. Using the rotation rates obtained by inversions of helioseismology, Komm et al. (2003) gave the maximum of $J$ is $5.5 \times 10^{37} \mathrm{~g} \mathrm{~cm} \mathrm{~s}^{-1}$ for the Michelson Doppler Image (MDI) data, or $5.32 \times 10^{37} \mathrm{~g} \mathrm{~cm} \mathrm{~s}^{-1}$ for the Global Oscillation Network Group (GONG) data near $r \approx 0.38 R_{\odot}$.

Model M3 is consistent with the Sun in the total solar angular momentum, the maximum of angular momentum density and the surface rotation rate. The magnetic fields are very important in the angular momentum transport in radiative interior. In our model, the core is rotating faster than the rest of the radiative interior. However, helioseismic results show the solar core is rotating more slowly than the rest of the radiative interior (Chaplin et al. 1999; Elsworth et al. 1995; Tomczyk et al. 1995), or else it is a solid-body rotation (Charbonneau et al. 1998). Some mechanism of angular momentum transport may be missed, or the value of $B_{r} / B$ should be larger than the one used in the core in our model. A gravity wave may be the candidate for angular momentum transport in the solar core, because the conditions for the known hydrodynamic instabilities occurrence are not satisfied in a core that is rotating slowly (Spruit et al. 1983).

\subsection{Mixing and its effects on the solar structure}

The radial profiles of hydrogen mass fraction $X$ are plotted in Fig. 2a. In Figs. 2b-2d, we compare the density, adiabatic index, and squared sound speed of our models with the 

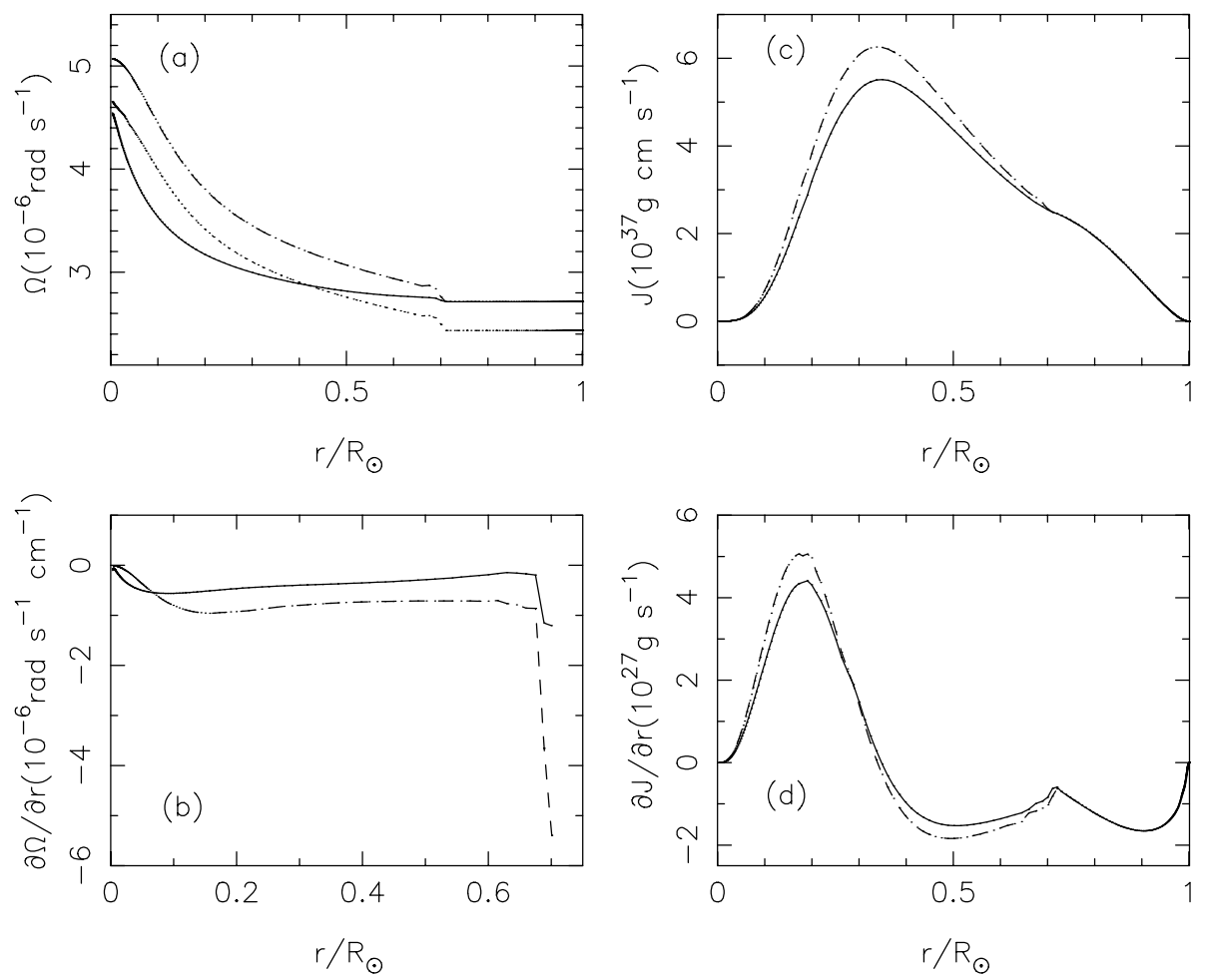

Fig. 1. Angular velocity $\Omega$, angular momentum density $J$, and their differentiation with respect to radius as a function of radius in the presentday Sun. a) The solid line is model M3 with total initial angular momentum $A=1.91 \times 10^{48} \mathrm{~g} \mathrm{~cm}^{2} \mathrm{~s}^{-1}$. The long-dashed line and the dotted line show model M2 with $A=2.1 \times 10^{48} \mathrm{~g} \mathrm{~cm}^{2} \mathrm{~s}^{-1}$ and $A=1.91 \times 10^{48} \mathrm{~g} \mathrm{~cm}^{2} \mathrm{~s}^{-1}$, respectively. b), c), and d) The solid line shows model M3 with $A=1.91 \times 10^{48} \mathrm{~g} \mathrm{~cm}^{2} \mathrm{~s}^{-1}$, and the long-dashed line is M2 with $A=2.1 \times 10^{48} \mathrm{~g} \mathrm{~cm}^{2} \mathrm{~s}^{-1}$.
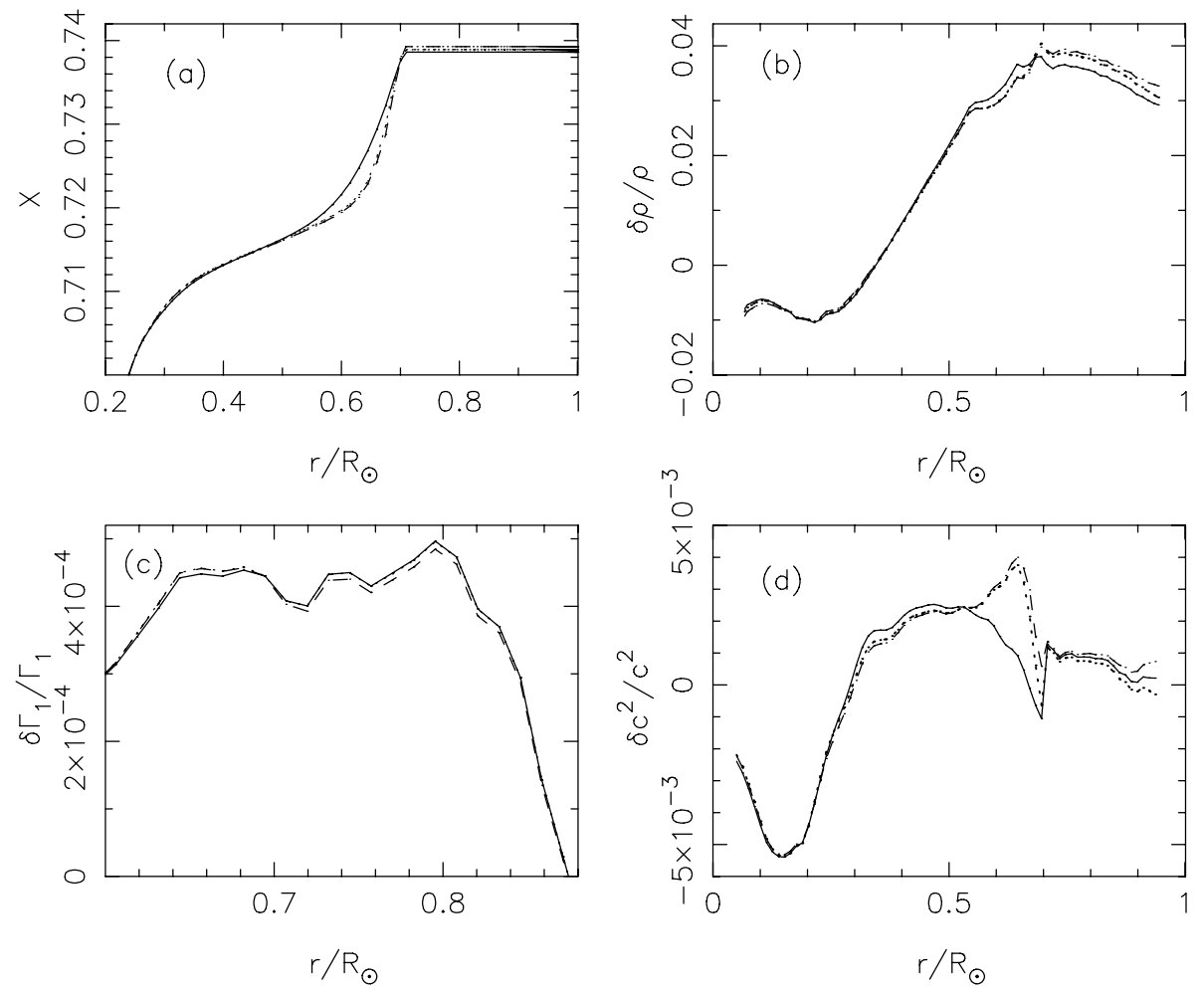

Fig. 2. a) Radial distribution of hydrogen mass fraction. b) Density difference between the Sun and the model. c) Adiabatic index difference between the Sun and the model. d) Squared sound-speed difference between the Sun and the model. All the differences are in the sense (Sun)(Model). The solid line refers to model M3. The long-dashed line refers to model M1. The dotted line refers to model M2 with total angular momentum $2.1 \times 10^{48} \mathrm{~g} \mathrm{~cm}^{2} \mathrm{~g}^{-1}$. 
helioseismic inversion results given by Basu et al. (1997c, 2000). The mixing of magnetic instabilities causes more of an increase of the hydrogen abundance just beneath the convective zone, but a decrease in the convective zone and near $r=0.3 R_{\odot}$, than that of model M1 and M2. The increase of $X$ between $0.5 R_{\odot}$ and $0.7 R_{\odot}$ causes a decrease in the mean molecular weight $\mu$ and density $\rho$, so that the difference in density $\rho$ increases in that region (Fig. 2b). Since the squared sound speed $c^{2} \propto T / \mu$, reducing $\mu$ must lead to an increase in $c^{2}$. The difference of squared sound speed, in the sense $\left(c_{\odot}^{2}-c_{\text {model }}^{2}\right) / c_{\text {model }}^{2}$, has a significant decrease, from 0.004 down to 0.0009 at $r=0.65 R_{\odot}$ (Fig. 2d), whereas the effect is the opposite between $0.3 R_{\odot}$ and $0.5 R_{\odot}$. In Fig. $2 \mathrm{~d}$, there is a negative value, $-1.06 \times 10^{-3}$, of $\delta c^{2} / c^{2}$ for models M2 and M3 at the bottom of the convective zone. That region is where the sharp radial change in $X$ happens due to the helium settling. The strong mixing leads to this negative value. The effect of mixing to the adiabatic index is very small, and the increase in $X$ causes a small increase in the adiabatic index (Fig. 2c).

Although material mixing can account for the bump in the sound-speed's squared difference between $0.6 R_{\odot}$ and $0.7 R_{\odot}$, the mass conservation causes a decrease in $X$, an increase in $\mu$, and hence an increase in $\delta c^{2} / c^{2}$ between $0.3 R_{\odot}$ and $0.5 R_{\odot}$. This change is not expected, but it must occur in a mixing model.

\section{Discussion and conclusions}

Parameter $f_{\Omega}$ is significantly less than unity in our model. This could be a consequence of overestimating the ratio of $B_{r}$ to $B$. The average ratio of the poloidal to toroidal field strength given by Fox \& Bernstein (1987) is $10^{-10}$. If we take a lower value for $B_{r} / B$, such as $10^{-6}$, instead of the previous one, we find that $f_{\Omega}$ is 1 order of magnitude. For simplicity, $f_{\Omega}$ and $f_{\mathrm{c}}$ were taken as constant so the assumption might not be strictly correct. If we take the parameter as $f_{\Omega}(r, t)$, then, the value of $f_{\Omega}(r, t)$ can be more than $6.5 \times 10^{-3}$ in most regions and times. But the uncertainty of magnetic $B_{r} / B$ is larger than that of $f_{\Omega}$. We take $f_{\Omega}$ as a constant. This is also a reason for low $f_{\Omega}$. The parameter $f_{\mathrm{c}}$ is less than unity, too. In the magnetic model, the angular momentum can be transported by both magnetic stresses and magnetohydrodynamical (MHD) instabilities, but only the MHD instabilities can mix material. So, the efficiency of material mixing must be less than that of angular momentum transport, i.e. low $f_{\mathrm{c}}$.

The magnetic field is more efficient than the secular shear instabilities in angular momentum transport outwards. Thus the rotation rate of model M3 is lower for $r<0.4 R_{\odot}$ and higher for $r>0.4 R_{\odot}$ than is model $\mathrm{M} 2$, which has the same total angular momentum as model M3. The model with the magnetic field is consistent with the heliosiesmic results in the solar total angular momentum, the maximum of angular momentum density, and the surface rotation rate.

In this paper, we obtained the diffusion coefficient of magnetic angular momentum transport, which depends on the radius, rotation rate, and magnetic fields. Using this diffusion coefficient, we find that the angular momentum can be efficiently redistributed by magnetic fields. The radio of $B_{r}$ to $B$ may be
$10^{-6}$ in the solar radiative interior. The coupling between the radiative region and convective zone can be strengthened by magnetic fields, hence the sharp gradient of rotation rote (Fig. 1b) is reduced at the bottom of the convective zone. In our model, the thickness of the layer of the sharp radial change in the rotation rate is about $0.036 R_{\odot}$, and the rotation is almost uniform in the radiative region. There is a maximum, $5.51 \times 10^{37} \mathrm{~g} \mathrm{~cm}^{2} \mathrm{~g}^{-1}$, of angular momentum density $J$ at $r=0.35 R_{\odot}$ for the model with a magnetic field. Material mixing associated with the angular momentum transfer leads to a change in the distribution of the chemical compositions, so that there is noticeable improvement in the profiles of the sound speed and density.

Acknowledgements. This work was supported by the NSFC through project 10473021, the National Key Fundamental Research Project G2000078401, and the Yunnan Science Foundation Council 2003A0027R.

\section{References}

Antia, H. M., Chitre, S. M., \& Thompson, M. J. 2000, A\&A, 360, 335 Alexander, D. R., \& Ferguson, J. W. 1994, ApJ, 437, 846

Bahcall, J. N., \& Pinsonneault, M. H. 1995, Rev. Mod. Phys., 67, 781

Barnes, G., Charbonneau, P., \& MacGregor, K. B. 1999, ApJ, 511, 466

Basu, S., \& Antia, H. 1997a, MNRAS, 287, 189

Basu, S. 1997b, MNRAS, 288, 572

Basu, S., Chaplin, W. J., Christensen-Dalsgaard, J., et al. 1997c, MNRAS, 292, 243

Basu, S., Pinsonneault, M. H., \& Bahcall, J. N. 2000, ApJ, 529, 1084

Braithwaite, J., \& Spruit, H. K. 2004, Nature, 431, 819

Brandenburg, A., \& Subramanian, K. 2005, Phys. Rep., 417, 1

Brun, A. S., Turck-Chieze, S., \& Zahn, J. P. 1999, ApJ, 525, 1032

Caligari, P., Moreno-Insertis, F., \& Schussler, M. 1995, ApJ, 441, 886

Chaboyer, B., \& Zahn, J.-P. 1992, A\&A, 253, 173

Chaboyer, B., Demarque, P., \& Pinsonneault, M. H. 1995, ApJ, 441, 865

Chaplin, W. J., Christensen-dalsgaard, J., Elsworth, Y., et al. 1999, MNRAS, 308, 405

Charbonneau, P., \& MacGregor, K. B. 1992, ApJ, 387, 639

Charbonneau, P., \& MacGregor, K. B. 1993, ApJ, 417, 762

Charbonneau, P., Tomczyk, S., Schou, J., \& Thompson, M. J. 1998, ApJ, 496, 1015

Charbonneau, P., Christensen-Dalsgaard, J., Henning, R., et al. 1999, ApJ, 527, 445

Charbonneau, P., \& MacGregor, K. B. 2001, ApJ, 559, 1094

Choudhuri, A. R., \& Gilman, P. A. 1987, ApJ, 316, 788

Christensen-Dalsgaard, J., Gough, D. O., \& Thompson, M. J. 1991, ApJ, 378, 413

Christensen-Dalsgaard, J., Dappen, W., Ajukov, S. V., et al. 1996, Science, 272, 1286

Christensen-Dalsgaard, J. 2002, RvMP, 74, 1073c

Corbard, T., Berthomieu, G., Provost, J., \& Morel, P. 1998, A\&A, 330, 1149

Corbard, T., Blanc-Féraud, L., Berthomieu, G., \& Provost, J. 1999, A\&A, 344, 696

Cowling, T. G. 1945, MNRAS, 105, 166

D'Silva, S., \& Choudhuri, A. R. 1993, A\&A, 272, 621

Dudorov, A. E., Krivodubsky, V. N., Ruzmajkina, T. V., \& Ruzmajkin, A. A. 1989 , AZh, 66, 809

Eggenberger, P., Maeder, A., \& Meynet, G. 2005, A\&A, 440, L9

Endal, A. S., \& Sofia, S. 1976, ApJ, 210, 184

Endal, A. S., \& Sofia, S. 1978, ApJ, 220, 279 
Elsworth, Y., Howe, R., Issak, G. R., et al. 1995, Nature, 376, 669

Fox, P. A., \& Bernstein, I. B. 1987, in The Internal Solar Angular Velocity, Reidel, ed. B. R. Durney, \& S. Sofia, Dordrecht, 213

Gough, D. O., Kosovichv, A. G., Toomre, J., et al. 1996, Science, 272, 1296

Grevesse, N., \& Sauval, A. J. 1998, in Solar Composition and Its Evolution: from Core to Corona, ed. C. Frohlich, M. C. E. Huber, S. K. Solanki, \& R. von Steiger (Dordrecht: Kluwer), 161

Huang, R. Q. 2004a, A\&A, 422, 981

Huang, R. Q. 2004b, A\&A, 425, 591

Iglesias, C., \& Rogers, F. J. 1996, ApJ, 464, 943

Kawaler, S. D. 1987, Pub. A.S.P., 99, 1322

Kawaler, S. D. 1988, ApJ, 333, 236

Kippenhahn, R., \& Thomas, H. C. 1970, in Stellar Rotation, ed. A. Slettebak (New York: Gordon and Breach), 20

Kippenhahn, R., \& Weigert, A. 1990, in Stellar Structure and Evolution (Berlin: Springer-Verlag), 431

Komm, R., Howe, R., Durney, B. R., \& Hill, F. 2003, ApJ, 586, 650

Kosovichev, A. G. 1996, ApJ, 469, L61

Langer, N. 1991, A\&A, 243, 155

Langer, N. 1992, A\&A, 256, L17

Maeder, A., \& Meynet, G. 1996, A\&A, 313, 140

Maeder, A., 1997, A\&A, 321, 134

Maeder, A., \& Zahn, J.-P. 1998, A\&A, 334, 1000

Maeder, A., \& Meynet, G. 2000, ARA\&A, 38, 43

Maeder, A., \& Meynet, G. 2003, A\&A, 411, 543

Maeder, A., \& Meynet, G. 2004, A\&A, 422, 225

MacDonald, J., \& Mullan, D. J. 2003, ApJ, 598, 560

Mestel, L. 1984, in 3rd Cambridge Workshop on Cool Stars, Stellar Systems, and the Sun, ed. S. L. Baliunas, \& L. Hartman (New York: Springer), 49
Mestel, L., Tayler, R. J., \& Moss, D. L. 1987, in The Internal Solar Angular Velocity, ed. B. R. Durney, \& S. Sofia (Dordrecht: Reidel), 143

Meynet, G., \& Maeder, A. 1997, A\&A, 321, 465

Moss, D. 1987, MNRAS, 226, 297

Paczyński, B. 1969, Acta. Astr., 19, 1

Paczyński, B. 1970, Acta. Astr., 20, 47

Parker, E. N. 1979, in Cosmical magentic fields: Their origin and their activity (Oxford: Clarendon Press)

Paternò, L. 1991, in Evolutionary Phenomena in the Universe, ed. P. Giannone, F. Melchiorri, \& F. Occhionero, Italian Physical Society Conference Proc., 32, 121

Pijpers, K. 1998, MNRAS, 297, L76

Pinsonneault, M. H., Kawaler, S. D., Sofia, S., \& Demarque, P. 1989, ApJ, 338, 424

Pitts, E., \& Tayler, R. J. 1986, MNRAS, 216, 139

Rogers, F. J., Swenson, J., \& Iglesias, C. 1996, ApJ, 456, 902

Schou, J., Antia, H. M., Basu, S., et al. 1998, ApJ, 505, 390

Spruit, H. C., Knobloch, E., \& Roxburgh, I. W. 1983, Nature, 304, 520

Spruit, H. C. 1999, A\&A, 349, 189

Spruit, H. C. 2002, A\&A, 381, 923

Thompson, M. J., Christensen-Dalsgaard, J., Miesch, M. S., \& Toomre, J. 2003, ARA\&A, 41, 599

Thoul, A. A., Bahcall, J. N., \& Loeb, A. 1994, ApJ, 421, 828

Tomczyk, S., Schou, J., \& Thompson, M. J. 1995, ApJ, 448, L57

Yang, J. Y., Li, Y., \& Xu, H. Y. 2001, A\&A, 366, 311

Zahn, J.-P. 1992, A\&A, 265, 115

Zahn, J.-P. 1993, in Astrophysical Fluid Dynamics, Les Houches XLVII, ed. J.-P. Zahn, \& Zinn-Justin (New York: Elsevier), 561 